\title{
Controle Robusto com Incerteza Paramétrica Para um Estabilizador de Sistema de Potência IMPLEMENTADO EM UM SISTEMA DE GERAÇÃO DE 10KVA
}

\author{
Cleyson A. Costa, Marcus Ciro Gomes, Fernando José Da Costa Jr., Walter Barra Jr., José A. L. \\ BARREIROS. \\ Laboratório de Controle de Sistema de Potência, Faculdade de Engenharia Elétrica, Universidade Federal do \\ Pará
}

Rua Augusto Corrêa, 01 - Guamá. CEP 66075-110. Caixa postal 479

\begin{abstract}
This work aimed to investigate experimentally robust control strategy, based on interval analysis, to dampen oscillations in a synchronous generator of $10 \mathrm{kVA}$. Since methods for identifying a discrete set of linear models (ARX) was obtained for different operating conditions, allowing to obtain a family of templates to design and analysis interval. For purposes of verifying the effectiveness of damping robust controller, it has been experimentally tested and their performance was compared to the performance of a controller damper fixed parameters. Tests response to the reference pulses applied to the automatic voltage regulator (AVR) on different conditions of operation of the system shown that the robust controller can maintain their specified performance while controlling the fixed damper has a marked deterioration in performance.
\end{abstract}

Keywords- Robust Control, Interval Analysis, Generation System Reduced Scale, Stability of Power System, Power System Stabilizer

Resumo-Este trabalho tem como objetivo investigar experimentalmente uma estratégia de controle robusta, baseado em análise intervalar, para amortecer oscilações eletromecânicas em um gerador síncrono de $10 \mathrm{kVA}$. A partir de métodos de identificação, um conjunto de modelos lineares discretos (ARX) foi obtido para diferentes condições de operação, permitindo a obtenção de uma família de modelos para uma análise e projeto intervalar. Para fins de verificação da efetividade de amortecimento do controlador robusto, o mesmo foi testado experimentalmente e seu desempenho foi comparado ao desempenho de um controlador amortecedor a parâmetros fixos. Testes de resposta a pulsos aplicados à referência do regulador automático de tensão (RAT) em variadas condições de operação do sistema demonstraram que o controlador robusto consegue manter o desempenho especificado, ao passo que o controlador amortecedor fixo apresenta uma acentuada degradação de desempenho.

Palavras-chave — Controle Robusto, Análise Intervalar, Sistema de Geração de Escala Reduzida, Estabilidade de Sistema de Potência, Estabilizador de Sistema de Potência.

\section{Introdução}

As oscilações eletromecânicas são fenômenos naturais que ocorrem no sistema de potência devido, muitas das vezes, a variações das condições de operação do gerador. Estas oscilações são nocivas ao sistema, pois reduzem a capacidade de transferência de potência, diminuem a vida útil das máquinas e reduzem a margem de estabilidade do sistema de potência. Visto isto, uma maneira prática de amortecer estas oscilações é a implementação de um controlador suplementar, que atua sobre a malha de controle de tensão do gerador, visando aumentar o amortecimento dos modos poucos amortecidos do sistema.

Quando os transitórios observados após perturbações no sistema se manifestam de forma oscilatória, estas oscilações deverão ser amortecidas caso o sistema seja estável na condição de operação considerada. $\mathrm{O}$ controlador incumbido de manter a estabilidade do sistema diante de oscilações eletromecânicas é denominado estabilizador de sistemas de potência (ESP), sua função é prover amortecimento adequado para os modos de oscilação eletromecânica dominantes no sistema.

Vários trabalhos de pesquisa têm sido realizados no sentido de buscar formas mais efetivas de projeto e estruturas de controladores para amortecer tais oscilações. Em [1] e [2] é apresentado um ESP digital para amortecer um modo de oscilação local observado em uma unidade geradora da Usina Hidrelétrica de Tucuruí, apresentando amortecimento superior que o ESP analógico instalado na mesma. Já em [3] um controlador com supervisor a ganhos programados fuzzy é utilizado para adaptar um controlador amortecedor em uma rede de controladores locais, apresentando um desempenho superior que um ESP convencional para uma faixa de condições de operação que um gerador pode assumir. Em [4] um ESP multibanda é projetado para atuar no amortecimento de diferentes modos de oscilação em testes realizado no sistema HydroQuébec.

O objetivo deste trabalho é investigar experimentalmente uma estratégia de controle robusta de alocação radial de polos baseado em análise intervalar para amortecer as oscilações eletromecânicas em um sistema de potência em escala reduzida de $10 \mathrm{kVA}$, pertencente a um sistema de geração do Laboratório de Controle de Sistemas de Potência (LACSPOT) da Universidade Federal do Pará.

Para o projeto dos controladores amortecedores testados procedeu-se a utilização de métodos de identificação, onde sete modelos lineares discretos (ARX) foram obtidos para condições de operação de 0,1 a 0,7 valores por unidade (pu) de potência ativa gerada, permitindo a obtenção de uma família de modelos e subsequente representação na forma intervalar para fins de projeto. 
Visando verificar a efetividade de amortecimento do controlador robusto sobre estudo, projetou-se, via alocação radial de polos, um controlador amortecedor local, a parâmetros fixos, para uma condição de operação específica do sistema de potência em escala reduzida. Em seguida, um conjunto de testes de resposta ao pulso aplicado na referência do regulador automático de tensão (RAT) em variadas condições de operação do sistema foi realizado. Os controladores robusto e local foram projetados para prover um amortecimento relativo de $\xi=0.3$ para o modo eletromecânico dominante.

\section{Apresentação do LACSPOT}

O Laboratório de Controle de Sistema de Potência (LACSPOT), da Faculdade de Engenharia Elétrica da Universidade Federal do Pará (UFPA), apresenta um sistema de geração em escala reduzida de $10 \mathrm{kVA}$, o qual é constituído por um gerador síncrono de polos salientes, um motor de corrente contínua (CC) de $9 \mathrm{~kW}$, um circuito indutivo para simular uma linha de transmissão e de um painel que contem toda instrumentação de automação, controle e acionamento do sistema. A Figura 1 apresenta uma imagem deste sistema.

O conjunto motor $\mathrm{CC}$ e gerador síncrono, denominado Micromáquina, apresenta um volante metálico de acoplamento entre o motor e o gerador para aumentar o momento de inércia das massas girantes do conjunto. Tal planta didática foi dimensionada de forma que seus parâmetros mecânicos e elétricos, em pu, fossem similares aos valores de um sistema de geração de grande porte [5].

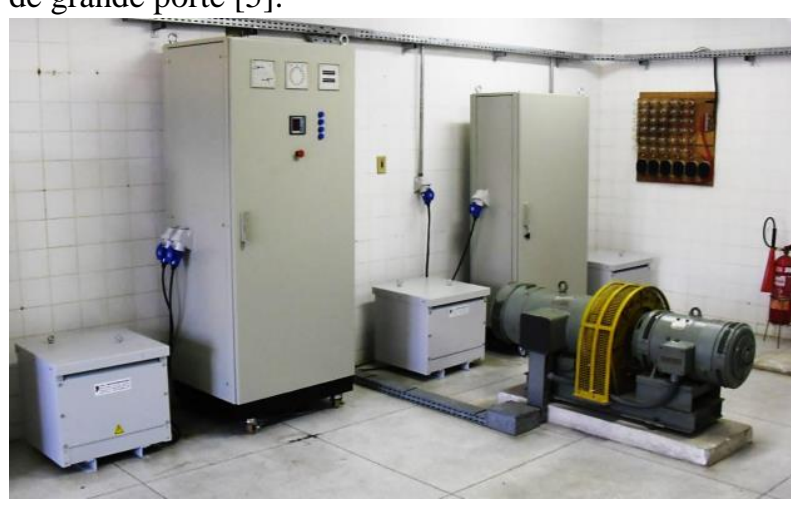

Figura 1. Sistema de geração em escala reduzida de 10kVA presente no LACSPOT.

\section{Estabilizador de Sistema de Potência}

A função básica de um ESP é adicionar amortecimento às oscilações eletromecânicas dominantes por meio de controle suplementar, fazendo uso de sinais auxiliares de estabilização.

A função de transferência do controlador amortecedor digital implementado neste trabalho é como apresentado na Figura 2, cuja entrada utilizada foi o desvio da potência ativa do gerador. Este canal de entrada foi selecionado por ser uma variável facilmente mensurável e que manifesta uma boa observabilidade em relação às oscilações eletromecânicas [6]. O sinal de saída do ESP é aplicado diretamente ao ponto de soma da referência do RAT, que é um controlador proporcional. A saída do estabilizador deve ser limitada para fins de não causar uma atuação excessiva sobre o campo da máquina e de modo a não intervir acentuadamente no controle de potência reativa.

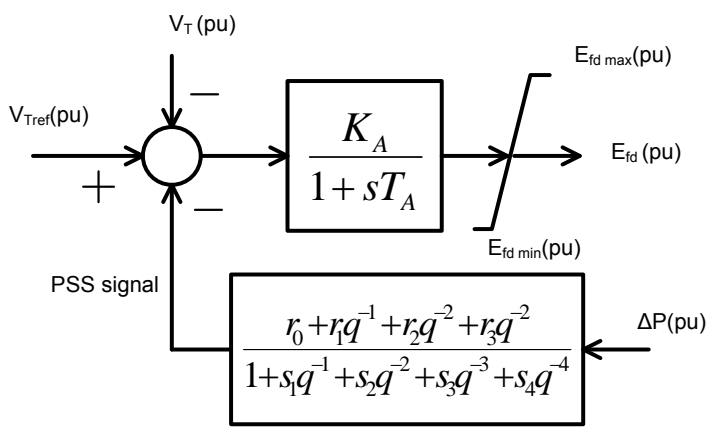

Figura 2. Estrutura do Estabilizador de Sistema de Potência Digital.

\section{Projeto do ESP Digital}

Na Figura 3, é apresentado o diagrama de bloco do sistema de um controle digital com a estrutura canônica RST. Tal estrutura permite o projeto de controladores digitais com a técnica polinomial de polos [7].

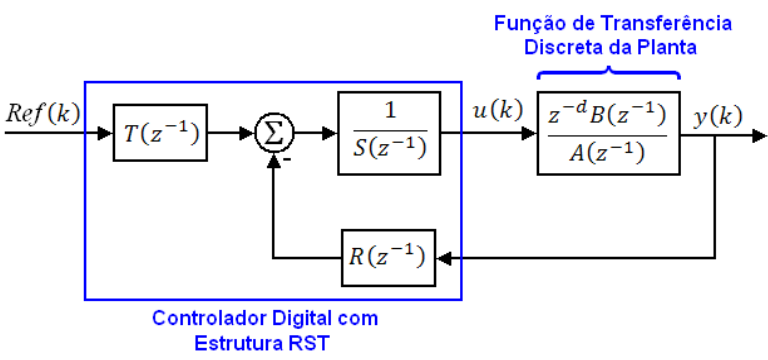

Figura 3. Diagrama de bloco do controlador digital RST.

Os polinômios do controlador devem ser expressos nas seguintes formas canônicas:

$$
\begin{gathered}
R\left(z^{-1}\right)=r_{0}+r_{1} z^{-1}+\ldots+r_{n r} z^{-n r}, \\
S\left(z^{-1}\right)=1+s_{1} z^{-1}+\ldots+s_{n s} z^{-n s}, \\
T\left(z^{-1}\right)=R\left(z^{-1}\right) \text { ou } R(1) .
\end{gathered}
$$

Foi selecionado $T\left(z^{-1}\right)=R\left(z^{-1}\right)$, pois não há interesse em desacoplar o rastreamento da regulação.

O modelo da planta discretizada deve obedecer à seguinte função de transferência:

$$
H\left(z^{-1}\right)=\frac{z^{-d} B\left(z^{-1}\right)}{A\left(z^{-1}\right)},
$$

onde d representa o atraso medido em múltiplos inteiros do período de amostragem.

O projeto de um controlador digital pelo método da alocação polinomial de polos consiste em resolver 
a equação diofantina para encontrar o valor dos parâmetros dos polinômios $\mathrm{R}$ e $\mathrm{S}$ do controlador. Expressa de forma matricial, a equação diofantina pode ser representada na forma de um sistema linear de equações algébricas, conforme a Equação (6).

$$
M x=P \text {, }
$$

em que $x$ é o vetor formado pelos parâmetros do controlador a ser calculado, e o vetor $P$ é formado pelos coeficientes do polinômio característico desejado. Sendo assim, os vetores $x$ e $P$ são representados pelas Equações (7) e (8), respectivamente.

$$
\begin{aligned}
& x=\left[1 s_{1} \ldots s_{N s^{\prime}} r_{0} r_{1} \ldots r_{N R^{\prime}}\right]^{T} \\
& P=\left[\begin{array}{lllll}
1 & P_{1} & P_{2} \ldots & \ldots & \ldots
\end{array}\right]^{T}
\end{aligned}
$$

A matriz M, chamada de matriz Silvestre, é definida segundo a Equação matricial (9):

$$
M=\left[\begin{array}{ccccccccc}
1 & 0 & \cdots & 0 & 0 & \cdots & \cdots & \cdots & 0 \\
a_{1} & 1 & & \vdots & \vdots & & & & \vdots \\
a_{2} & a_{1} & & 0 & 0 & & & & \vdots \\
a_{2} & a_{2} & & 1 & \hat{b}_{1} & & & & 0 \\
\vdots & a_{a} & & a_{1} & \vdots & & & & \hat{b}_{1} \\
a_{n_{A}} & \vdots & & a_{2} & \hat{b}_{n_{B}} & & & & \hat{b}_{2} \\
0 & & & a_{2} & 0 & & & & \hat{b}_{a} \\
\vdots & & \vdots & \vdots & \vdots & \vdots & & \vdots & \vdots \\
0 & \cdots & 0 & a_{n_{A}} & 0 & 0 & \cdots & 0 & \hat{b}_{n_{B}}
\end{array}\right]
$$

Um caso particular do método de alocação polinomial de polos é o caso em que os polos dominantes devem ser deslocados radialmente para uma posição mais interior no círculo unitário do plano z. Isto corresponde uma posição com melhor margem de estabilidade. Este caso é denominado de Alocação Radial de Polos (do inglês, pole shifting).

No presente projeto, o polinômio desejado é obtido aplicando-se um fator de contração $\alpha \quad(0<\alpha<1)$ aos polos dominantes, conforme mostrado na Equação (10). Os demais polos de $P\left(z^{-1}\right)$ são alocados na origem.

$$
P\left(z^{-1}\right)=1+\alpha a_{1} z^{-1}+\ldots+\alpha^{n A} a_{n A} z^{-n A}
$$

O fator de contração $\alpha$ é especificado pelo projetista e está intimamente relacionado ao coeficiente de amortecimento desejado para o sistema em malha fechada, ao coeficiente de amortecimento do sistema em malha aberta e ao intervalo de amostragem selecionado. $\mathrm{O}$ fator $\alpha$ pode ser calculado utilizando-se a Equação (11):

$$
\alpha=e^{-\left(\xi_{d}-\xi_{n}\right) \omega_{n} T_{s}}
$$

onde $\xi_{d}$ e $\xi_{n}$ é o coeficiente de amortecimento desejado e o coeficiente de amortecimento natural do sistema, respectivamente.

Portanto, a equação de Bezout assume a forma:

$$
P\left(z^{-1}\right)=A\left(z^{-1}\right) S\left(z^{-1}\right)+B\left(z^{-1}\right) R\left(z^{-1}\right),
$$

a qual pode ser resolvida por meio de sua forma matricial mostrada na Equação (6).

$$
\left[\begin{array}{cccccccc}
1 & 0 & \cdots & 0 & b_{1} & \cdots & \cdots & 0 \\
a_{1} & 1 & & 0 & b_{2} & b_{1} & & 0 \\
\vdots & a_{1} & & \vdots & \vdots & b_{2} & & \vdots \\
a_{n_{A}} & \vdots & & 1 & b_{n_{B}} & \vdots & & b_{1} \\
0 & a_{n_{A}} & & a_{1} & 0 & b_{n_{B}} & & b_{2} \\
\vdots & 0 & & \vdots & \vdots & 0 & & \vdots \\
\vdots & \vdots & & \vdots & \vdots & \vdots & & \vdots \\
0 & 0 & \cdots & a_{n_{A}} & 0 & 0 & \cdots & b_{n_{B}}
\end{array}\right]\left[\begin{array}{c}
s_{1} \\
s_{2} \\
\vdots \\
\vdots \\
s_{n_{S}} \\
r_{0} \\
r_{1} \\
\vdots \\
r_{n_{R}}
\end{array}\right]=\left[\begin{array}{c}
(\alpha-1) a_{1} \\
\left(\alpha^{2}-1\right) a_{2} \\
\vdots \\
\vdots \\
\left(\alpha^{n_{A}}-1\right) a_{n_{R}} \\
0 \\
\vdots \\
0
\end{array}\right]
$$

\section{Projeto do ESP Robusto Intervalar}

No projeto do ESP intervalar, a planta é representada por uma função de transferência cujo numerador e o denominador são polinômios com coeficientes descritos por intervalos fechados [8], conforme definido pela Equação (14):

$$
H^{\prime}\left(z^{-1}\right)=\frac{z^{-d}\left[B^{\prime}\left(z^{-1}\right)\right]}{\left[A^{\prime}\left(z^{-1}\right)\right]},
$$

sendo,

$$
\begin{gathered}
{\left[B^{\prime}\left(z^{-1}\right)\right]=\left[b_{0}\right]+\left[b_{1}\right] z^{-1}+\ldots+\left[b_{n B}\right] z^{-n B},} \\
{\left[A^{\prime}\left(z^{-1}\right)\right]=1+\left[a_{0}\right] z^{-1}+\ldots+\left[a_{n A}\right] z^{-n A} .}
\end{gathered}
$$

Por definição:

$$
\begin{aligned}
& {\left[b_{n}\right]=\left[b_{n}^{-}, b_{n}^{+}\right],} \\
& {\left[a_{n}\right]=\left[a_{n}^{-}, a_{n}^{+}\right],}
\end{aligned}
$$

onde $b_{n}^{-}$e $a_{n}^{-}$são os limites inferiores, $b_{n}^{+} \mathrm{e}$ $a_{n}^{+}$são os limites superiores dos coeficientes $b_{n} \mathrm{e}$ $a_{n}$, respectivamente.

Na Tabela 1, são apresentados os valores dos coeficientes intervalares, obtidos a partir de identificação paramétrica com entrada exógena (ARX) [9], [10], identificados para setes pontos de operação distintos, que correspondem a sete níveis de geração de potência ativa variando no intervalo de $[0,1 ; 0,7] \mathrm{pu}$.

Tabela 1. Valores intervalares dos parâmetros da planta para sete pontos de operação $[0,1 ; 0,7] \mathrm{pu}$.

\begin{tabular}{c|c}
\hline Coeficiente & Valor \\
\hline$b_{0}$ & {$[0.0,0.0]$} \\
\hline$b_{1}$ & {$[0.16334,0.30183]$} \\
\hline$b_{2}$ & {$[-0.69765,-0.37944]$} \\
\hline$b_{3}$ & {$[0.03082,0.14706]$} \\
\hline$b_{4}$ & {$[0.09122,0.22995]$} \\
\hline$a_{1}$ & {$[-2.50550,-2.27102]$} \\
\hline$a_{2}$ & {$[2.46117,2.83226]$} \\
\hline$a_{3}$ & {$[-1.70762,-1.49321]$} \\
\hline$a_{4}$ & {$[0.51469,0.54816]$} \\
\hline \multicolumn{2}{|c}{}
\end{tabular}

As incertezas de natureza intervalar nos coeficientes dos polinômios $\left[B^{\prime}\left(z^{-1}\right)\right]$ e $\left[A^{\prime}\left(z^{-1}\right)\right]$ resultam con- 
sequentemente em uma matriz de Silvester intervalar. $\mathrm{O}$ vetor que representa os polos dominantes de malha fechada também é representado por um vetor de coeficientes intervalares, como mostrado na Equação (19):

$$
\left[P\left(z^{-1}\right)\right]=1+[\alpha]\left[a_{1}\right] z^{-1}+\ldots+\left[\alpha^{n A}\right]\left[a_{n A}\right] z^{-n A},
$$

sendo $[\alpha]$ o coeficiente de contração com incertezas intervalar.

A equação diofantina associada à matriz de Silvester intervalar e ao vetor $\left[P\left(z^{-1}\right)\right]$ é uma equação algébrica intervalar, cujo conjunto solução é um controlador com parâmetros intervalares capaz de garantir o desempenho desejado em malha fechada do sistema com incerteza paramétrica.

A partir dos dados da Tabela 1, é projetado o controlador intervalar seguindo os mesmos procedimentos adotados na seção 4 e considerando-se os mesmos requisitos em forma de coeficiente de amortecimento. Na Tabela 2, são apresentados os valores dos coeficientes do controlador amortecedor intervalar obtidos a partir do uso da ferramenta INTLAB/MATLAB [11].

Tabela 2 Controlador intervalar para um coeficiente de amortecimento relativo desejado de 0,3 .

\begin{tabular}{c|c}
\hline Coeficiente & Valor \\
\hline$r_{0}$ & {$[0.11355,012527]$} \\
\hline$r_{1}$ & {$[-252.83,253.01]$} \\
\hline$r_{2}$ & {$[-459.28,459.01]$} \\
\hline$r_{3}$ & {$[-243.77,243.63]$} \\
\hline$s_{1}$ & {$[-1992.55,1993.58]$} \\
\hline$s_{2}$ & {$[-1416.34,1415.58]$} \\
\hline$s_{3}$ & {$[-787.50,787.97]$} \\
\hline
\end{tabular}

Para implementação prática, foi selecionado o controlador central de acordo com [8], pois o controlador robusto intervalar foi implementado na forma de um controlador a parâmetros fixos. Neste caso, o valor de cada paramento foi o valor central do correspondente intervalo da Tabela 2.

\section{Resultados Experimentais Obtidos}

Para a realização dos testes do ESP digital no sistema microgerador de 10kVA, o sistema de geração foi colocado em operação e, com auxílio do conjunto sincronoscópio, realizou-se o paralelismo do grupogerador com a rede elétrica. Foram registradas as curvas de resposta ao pulso de 0.07 pu e $100 \mathrm{~ms}$ de duração, aplicado no somador localizado na entrada do RAT para uma situação sem o estabilizador, com o ESP local projetado para um ponto de operação de 0.4 pu de potência ativa e zero de reativa, e para o ESP robusto intervalar. Na Figura 4, é ilustrado um diagrama esquemático da conexão do ESP ao processo.

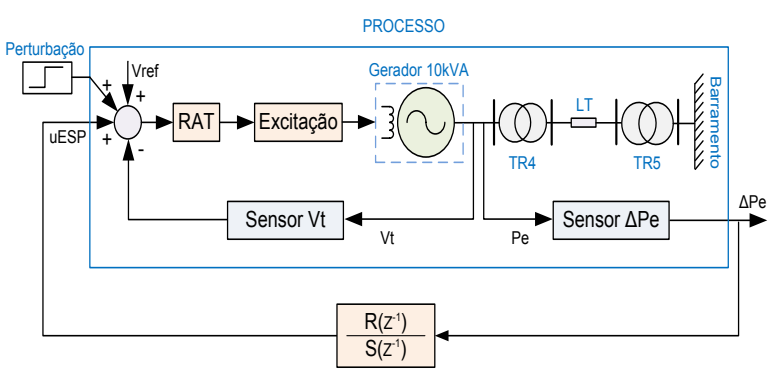

Figura 4. Diagrama esquemático do ensaio experimental.

Os ensaios para testar o funcionamento do ESP digital foram realizados para quatro pontos de operação diferentes. O Primeiro ponto foi: Potência Ativa $(P)=0.2 p u$ e Potência Reativa $(Q)=0$ e o registro da forma de onda do desvio da potência ativa para um pulso de $7 \%$ na entrada de perturbação está mostrado na Figura 5.

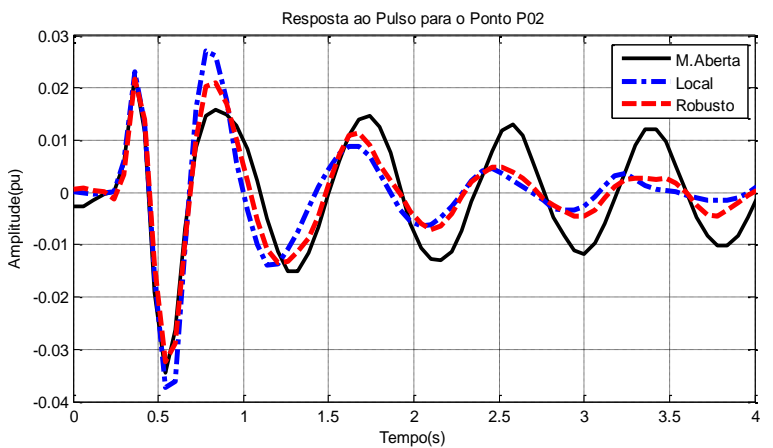

Figura 5. Resposta ao pulso de $7 \%$ para $(\mathrm{P}=0.2 \mathrm{pu}$ e $\mathrm{Q}=0)$.

Para o segundo ensaio, alterou-se o ponto de operação para: $P=0.4 p u$ e $Q=0$ e realizou-se a aplicação do pulso de $7 \%$ de perturbação. Na Figura 6 , é mostrada a resposta ao pulso para este ponto de operação.

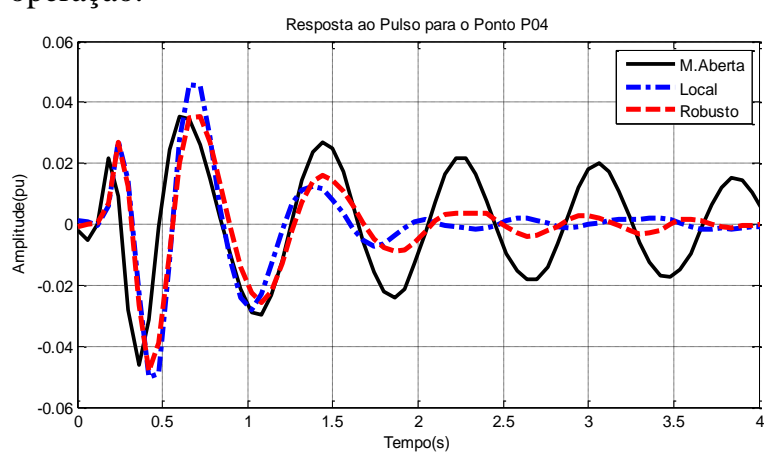

Figura 6. Resposta ao pulso de $7 \%$ para $(\mathrm{P}=0.4 \mathrm{pu}$ e $\mathrm{Q}=0)$

Para o terceiro ensaio, alterou-se o ponto de operação para: $P=0.6 p u$ e $Q=0$ e realizou-se a aplicação do pulso de $7 \%$ de perturbação. Na Figura 7 , é mostrada a resposta ao pulso para este ponto de operação. 


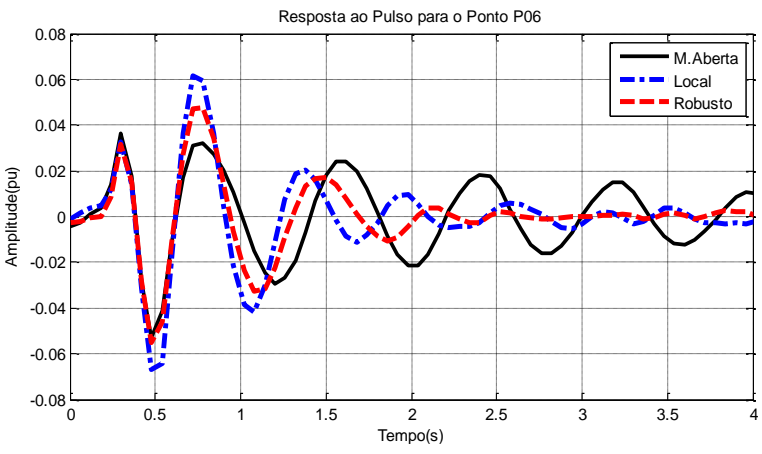

Figura 7. Função ISE para o desvio de potência elétrica.

Por fim, no quarto ensaio, alterou-se o ponto de operação para: $P=0.7 p u$ e $Q=0$ e realizou-se a aplicação do pulso de $7 \%$ de perturbação como nos três procedimentos anteriores. Na Figura 8, é mostrada a resposta ao pulso para este ponto de operação.

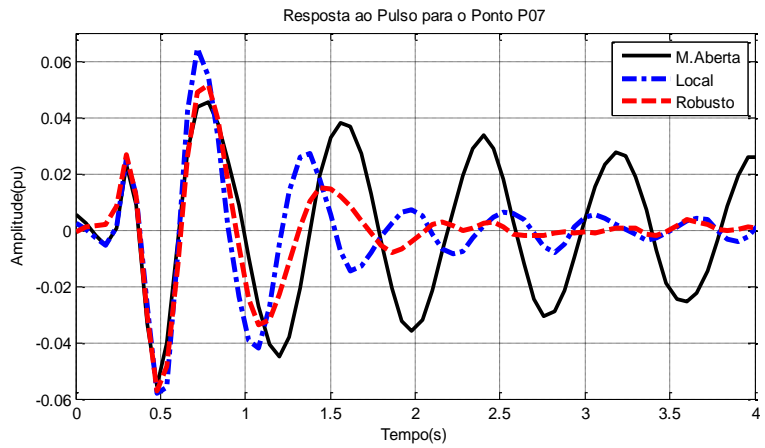

Figura 8. Resposta ao pulso de $7 \%$ para $(\mathrm{P}=0.7 \mathrm{pu}$ e $\mathrm{Q}=0)$.

Para analisar o custo beneficio dos controladores implementados, foi calculador a função custo segundo o critério da integral do erro quadrático ISE ("Integral square error"), conforme a Equação (19). O índice ISE foi calculado tanto para o desvio de potência ativa quanto para o esforço de controle do ESP.

$$
J_{I S E}=\int_{0}^{t} e^{2}(t) d t
$$

Na Figura 9, são apresentados os valores do ISE para o desvio de potência elétrica (dPe), em relação a cada ponto de operação que foi realizado os ensaios de perturbação do tipo pulso. Na figura, são mostrados os valores do ISE para o controlador intervalar e do controlador fixo, observa-se que em todos os pontos o valor do ISE para o controlador intervalar é sempre menor que o controlador fixo, comprovando que o controlador robusto tem melhor desempenho no amortecimento das oscilações eletromecânicas.

Na Figura 10, são apresentados os correspondentes valores do ISE calculados para o sinal de controle do ESP fixo e do ESP intervalar (Ue), em relação a cada ponto de operação. Observa-se, novamente, que em todos os pontos o valor do ISE para o sinal de controle do controlador intervalar é sempre menor que o sinal de controle do controlador fixo. Este resultado indica que um melhor desempenho do ESP robusto foi obtido com menor esforço de controle, quando comparado ao esforço de controle do ESP fixo.

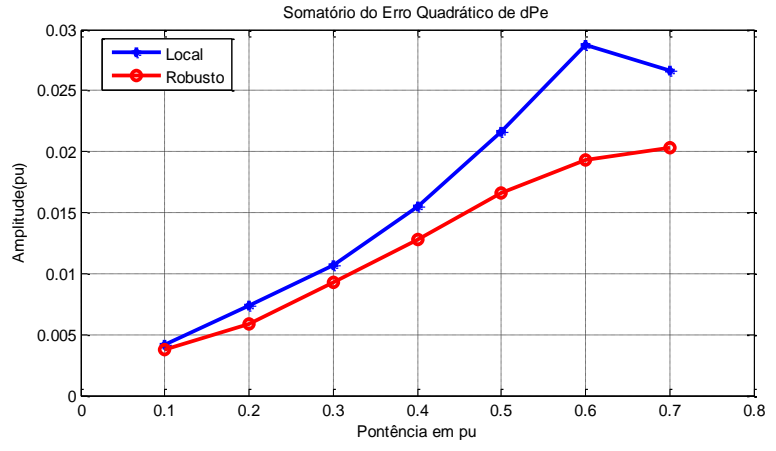

Figura 9. Função ISE para o desvio de potência elétrica.

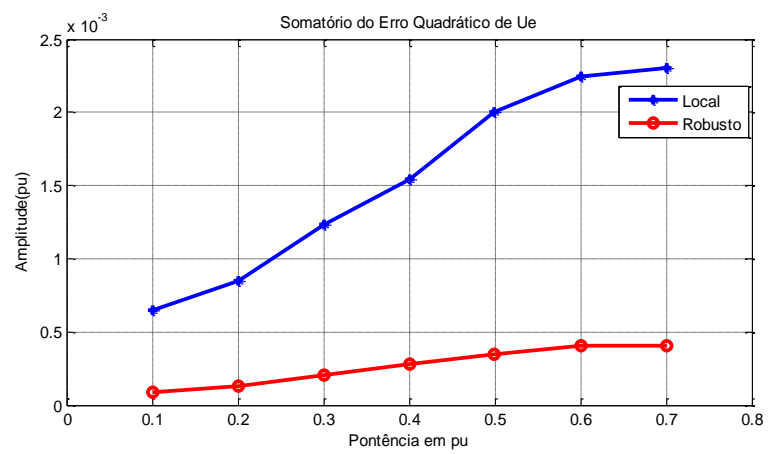

Figura 10. Função ISE para o desvio do sinal de controle do ESP.

\section{Conclusão}

Neste trabalho, foi apresentado um método de sintonia dos parâmetros de um controlador amortecedor adotada para projetar um controlador por alocação robusta de polos, considerando-se incertezas paramétricas do tipo intervalar, referente a variação do ponto de operação do sistema, cujo modelo matemático foi obtido através de técnicas de identificação paramétricas.

Testes de resposta ao impulso aplicado na referência do regulador automático de tensão (RAT) em variadas condições de operação do sistema demonstraram que o controlador robusto intervalar consegue manter o desempenho mais próximo de um amortecimento especificado, ao passo que o controlador amortecedor fixo evidenciou acentuada dessintonia para pontos de operação diferentes do seu ponto de projeto.

\section{Agradecimentos}

Os autores agradecem a Universidade Federal do Pará pelo ensino e espaço concedido e ao CNPq, CAPES e Eletronorte, pelo financeiro recebido por meio dos projetos coordenados pelos Professores: Dr. Augusto L. Barreiros, Dr. Carlos T. da Costa Jr. e Dr. Marcus V. A. Nunes.

\section{Referências Bibliográficas}

[1] Lana, J. J. ; Nogueira, F. G.; Costa Junior, C. T.; Barra Junior, W.; Barreiros, J. A. L.; Silva, O. F.; 
Gomes, M. C. M.; Moraes, A. R. B.. Projeto E Implementação De Um Estabilizador De Sistema De Potência Digital Aplicado À Uma Unidade Geradora Da Usina De Tucuruí. In: XX Seminário Nacional de Produção e Transmissão de Energia Elétrica snptee, 2009, recife. xx snptee seminário nacional de produção e transmissão de energia elétrica, 2009.

[2] Nogueira, F. G. ; Barra Junior, W.; Costa Jr., Carlos T. ; Barreiros, J. A. L. ; Moraes, A. R. B. ; Nascimento Filho, P. S. ; Gomes, M. C. M. ; Lana, J. J.. Estabilizador de sistemas de potencia digital aplicado a uma unidade geradora da UHE de Tucurui. Controle \& Automação (Impresso), v. 22, p. 535-544, 2011.

[3] Barra Junior, Nogueira, F. G.,W.; Nascimento Filho, P. S.; Moraes, A. R. B. ; Gomes, M. C. M.; Costa Junior, C. T.; Barreiros, J. A. L.; Campos, R. ; Lana, J. J. Projeto de um Estabilizador de Sistemas de Potência Digital com Adaptação Paramétrica Baseada em Lógica Fuzzy para uma Unidade Geradora de 350 MVA. In: ninth latinamerican congress on electricity generation and transmission - clagtee 2011, 2011, mar del plata. anais of 9th latin-american congress on electricity generation and transmission - clagtee 2011, 2011.

[4] Kamwa, I., R. Grondin, And G. Trudel,. IEEE PSS2B Versus PSS4B: The Limits of Performance of Modern Power System Stabilizers. IEEE transactions on power systems, vol. 20, no. 2, may 2005. DOI: 10.1109/TPWRS.2005.846197

[5] De.Faria, F.P., De Moraes, A. R. B. Nascimento Filho, P. S., Costa, C. A., Sousa, M. R. B., Ayres Jr., F. A. de C., Da Costa Jr., F. J., Barra Jr., Walter, Da Costa Jr., C. T., Barreiros, J. A. L., Nunes, M. V. A. Modernização da Instrumentação para Controle e Acionamento de um Sistema de Geração em Escala Reduzida. Congresso Brasileiro de Automática -2012 .

[6] De Moraes, A. R. B., 2011. Desenvolvimento e Implementação de Estratégia de Controle Digital para Regulação de Tensão e Amortecimento de Oscilações Eletromecânica em um Gerador Síncrono de 10KVA. Belém: Universidade Federal do Pará.

[7] Landau, I.D.; Zito, G. Digital Control Systems: Design, Identification and Implementation. Germany: Springer, 2006.

[8] Lordelo, Alfredo Del Sole. Análise e Projeto de controladores robustos por alocação de polos via análise intervalar. Tese de Doutorado. Campinas, 2004.

[9] Aguirre, L. A.. Introdução à Identificação de Sistemas: técnicas lineares e não-lineares aplicadas a sistemas reais. 3. Ed. Rev. e ampl.Belo Horizonte: Editora UFMG, 2007.

[10] Coelho, A. A. R.. Identificação de sistemas dinamicos lineares. Florianópolis Ed. Da UFSC, 2004.

[11] S.M. Rump. INTLAB - INTerval LABoratory. In Tibor Csendes, editor, Developments in Reliable
Computing, pages 77-104. Kluwer Academic Publishers, Dordrecht, 1999. DOI: 10.1007/978-94-017-1247-7_7

[12] Kundur, P. Power System Stability and Control. New York: McGraw-Hill, Inc, 1994.

[13] Sauer, P.W.; Pai, M.A. Power System Dynamics and Stability. Rio de Janeiro: Prentice Hall, 1998.

[14] Kimbark, E. W. Power System Stability: Synchronous Machines. New York: Dover Publications, Inc, 1968.

[15] Saadat, H. Power System Analysis. McGraw-Hill Series in Electrical and Computer Engineering, 2002. 